\title{
KARTENBEILAGE
}

\author{
ז" \\ G. Schütte
}

\section{die quellen der ptolemäischen karten von Nordeuropa}

(s. 1-46)

\section{VORBEMERKUNG.}

Die gelegenheit zum studium der photographien nach verschiedenen ptolemäischen atlashandschriften verdanke ich, wie schon auf S. 46 erwähnt, dem gütigen entgegenkommen des herrn professor Jos. Fischer S.J. in Feldkirch, der auch immer bereit war, mir durch erklärungen und berichtigungen beizustehen.

Die bei den meisten karten benutzten clichés entstammen meinen veröffentlichungen in den zeitschriften der geographischen gesellschaften zu Edinburgh und Kopenhagen 1914-15 und sind mir von den genannten gesellschaften freundlichst $z u$ gebote gestellt worden.

Verschiedene zeichnungen sind seit den ersten veröffentlichungen in dem "Saga Book " der "Viking Society" und in "The Scottish Geographical Magazine " mehr oder weniger stark umgeändert worden; so namentlich die übersichtskarte ("Scott. Geogr. Mag." 1914 , s. 297) und die karten der vorlagen Aa, Ac \& Ad, und $C$, sowie fig. 26 . Die zeichnung der vorlage $D$ ist zurückgezogen worden, vgl. oben S. 36, "Saga-Book" 1913, vol. VIII, part. I, s. 77, und "Scott. Geogr. Mag." 1914, s. 71 .

Weitere verbesserungen habe ich nicht unternehmen können, da meine bisherigen veröffentlichungen wie gesagt noch nirgends kritisiert wurden.

Die wahrscheinlichen doubletten Trabana (Cod. Vatic. 191) und Tabana, die laut fig. 1 beziehungsweise den vorlagen $E$ und $F$ überwiesen sind, möchte ich jetzt eher umgekehrt verteilen, s. fig. 24-26. Fig. 1 war aber bereits reproduciert, bevor ich diese anderung unternahm. Zermizegethuza fig. 1 sollte Zarmizegethusa heissen. Etwaige ungenauigkeiten der alteren zeichnungen konntc ich nicht bessern ohne ganz neue cliches herzustellen. 


\section{NHALT}

Fig. 1. Ptolemäische vorlagen in Nord-und Mitteleuropa. Allgemeine übersicht. $\$ 7 \ldots$ III

"2-4. Germania, Chersonesus Cimbrica, Scandia. $\$ 8$

IV-VI

VII

VIII-IX

„8-11. Südwestgermanien mit Limes Transrhenanus; section der Tabula Peutingeriana; vorlage Ab. $\$ 10 \ldots \ldots \ldots \ldots \ldots \ldots \ldots \ldots$

"12-18. Dacia; section der Tabula Peutingeriana;
vorlagen Ac, Ad, Ae. $\$ 11 \ldots \ldots \ldots \ldots \ldots$

"19-20. Ostgermanien; vorlagen B1 \& B2. $\$ 12 \ldots$

"21-23. Belgien u. Nordwestgermanien; section der Tabula Peutingeriana; vorlage C. $\$ 13$

$$
\mathrm{X}-\mathrm{XIII}
$$

XIV-XIX

$\mathrm{XX}-\mathrm{XXI}$

XXII-XXIV

" 24-26. Nordostgermanien u. Sarmatien; vorlagen E \& F. $\$ 15 \ldots \ldots \ldots \ldots \ldots \ldots \ldots \ldots \ldots \ldots \ldots$ XX $\ldots \ldots$ XVII

"27-28. Scandia; vorlage Sk. $\$ 16 \ldots \ldots \ldots \ldots \ldots$ XXVIII-XIX

Fig. 2, 6, 8, 12 enthalten reproductionen oder zeichnungen nach dem Cod. Urbinas 82 IV, VIII, X, XVI

" 3 enthält eine reproduction nach dem Cod. Burney $111 \ldots \ldots \ldots \ldots \ldots \ldots \ldots \ldots \ldots$ v

" 28 - $\quad$ Cod. Athous Vatopediensis .. XXIX 


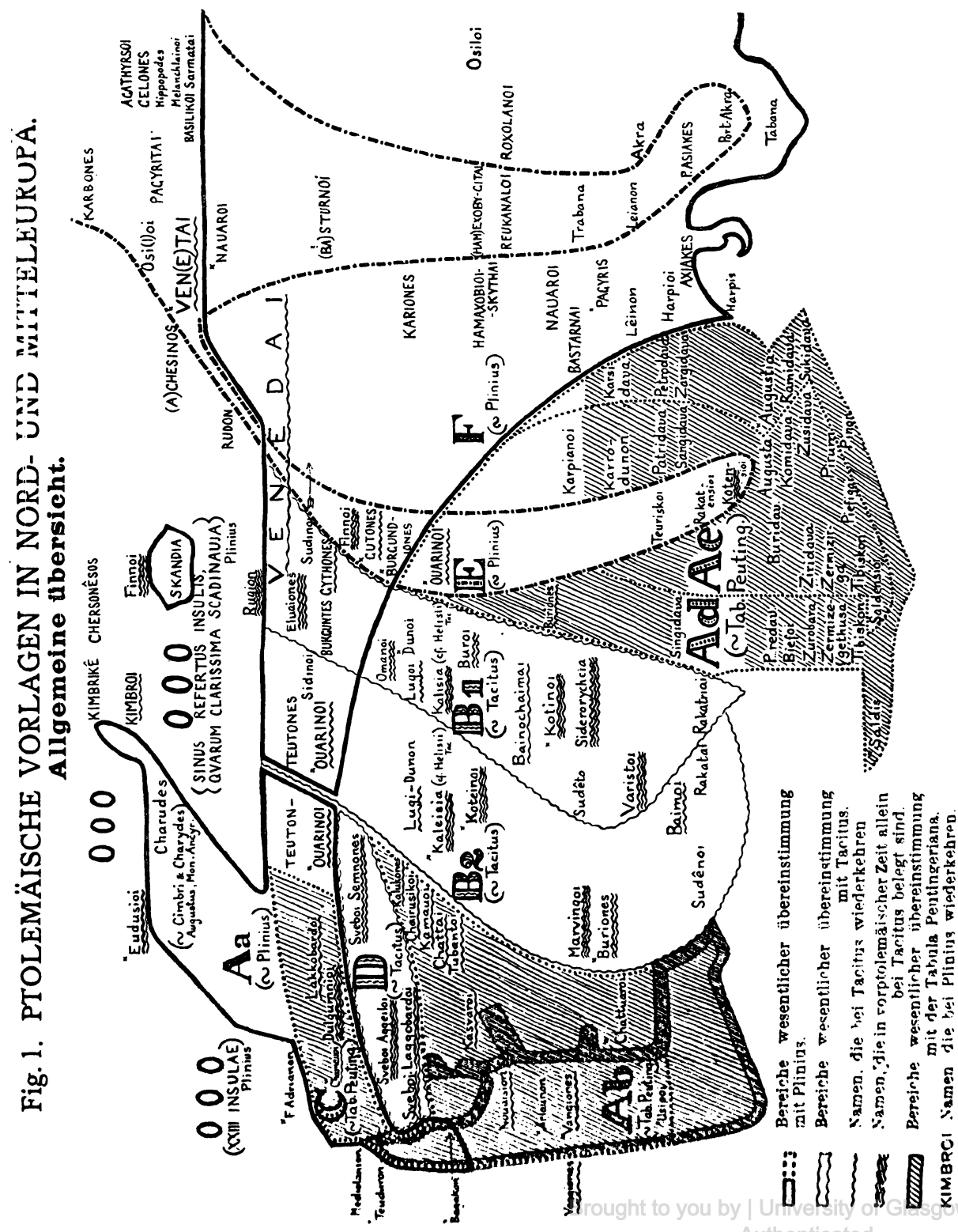


Fig. 2.

\section{GERM ANIA, CHERSONESUS CIMBRICA, SCANDIA. Ausgabe $A$.}

nach dem Codex Urbinas 82 der Bibl. Vaticana. 13. jahrhundert.

Mit erlauhnis vem prof. Jos. Fischer. S. J. S. \$ 9.

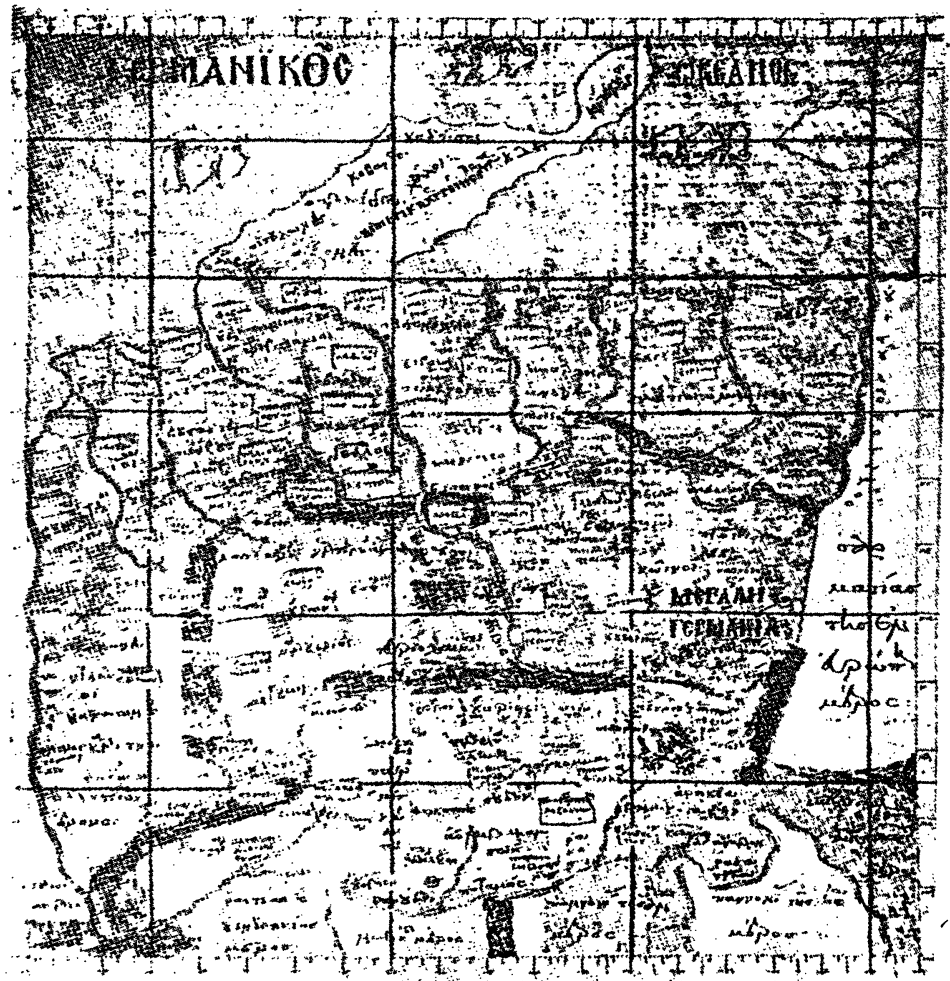


Fig. 3.

\section{GERMANIA, CHERSONESUS CIMBRICA, SCANDIA. Ausgabe B.}

nach dem Codex Burney 111 im British Musæum, 13. jahrhundert.

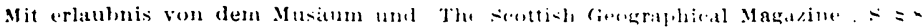

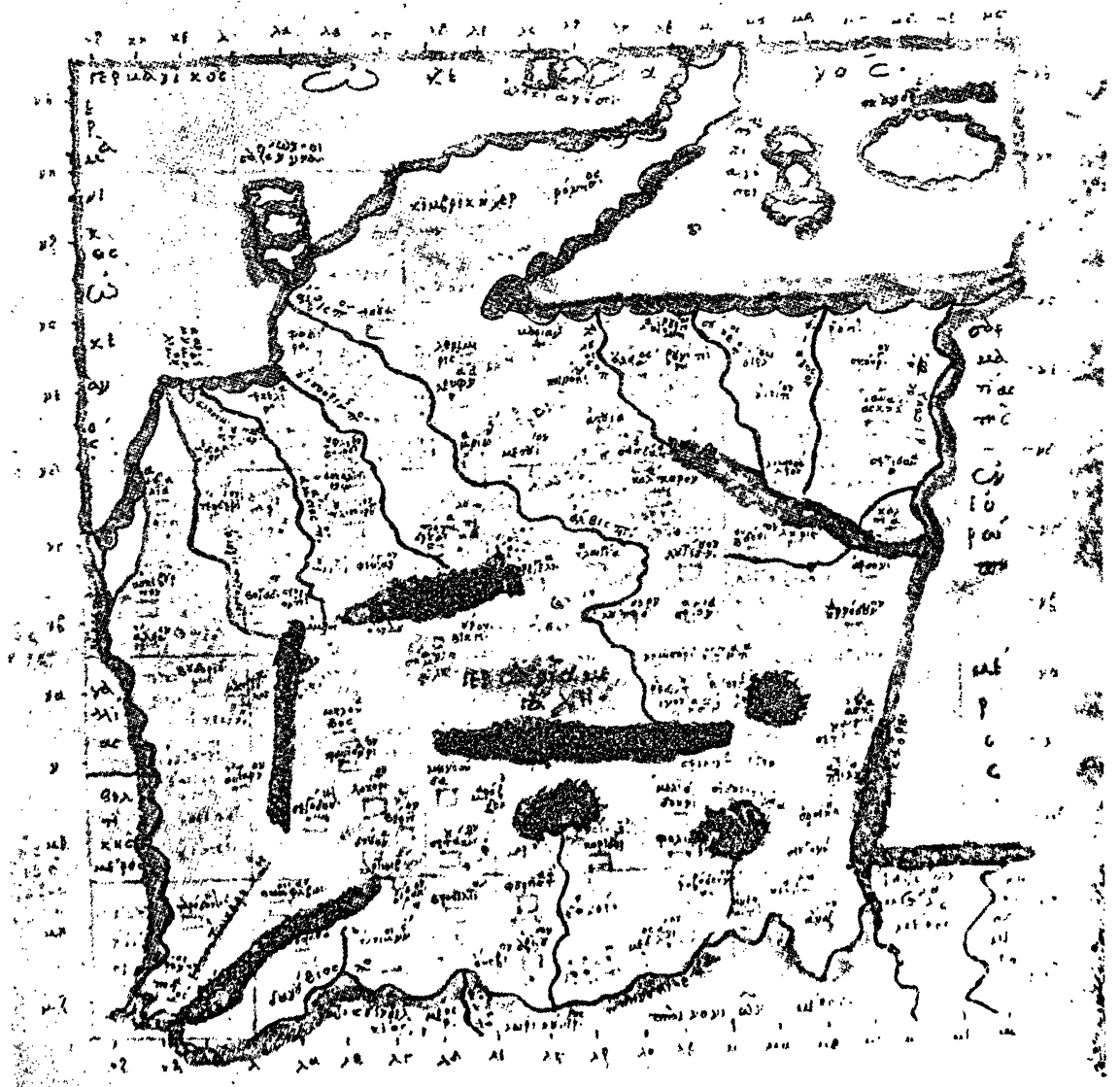


Fig. 4.

GERMANIA, CHERSONESUS CIMBRICA, SCANDIA. Typus der römischen ausgaben nach dem Codex Ebnerianus (lat.) in dem New York Public Library gezeichnet von Nicolaus Donis, 15. jahrhundert.

Mit erlaubuis von prof. Jos. Fiselher s. J hach ('atolic Historical Records and studiese, New lork, 19. \&. 222-228. \&. s. 8.

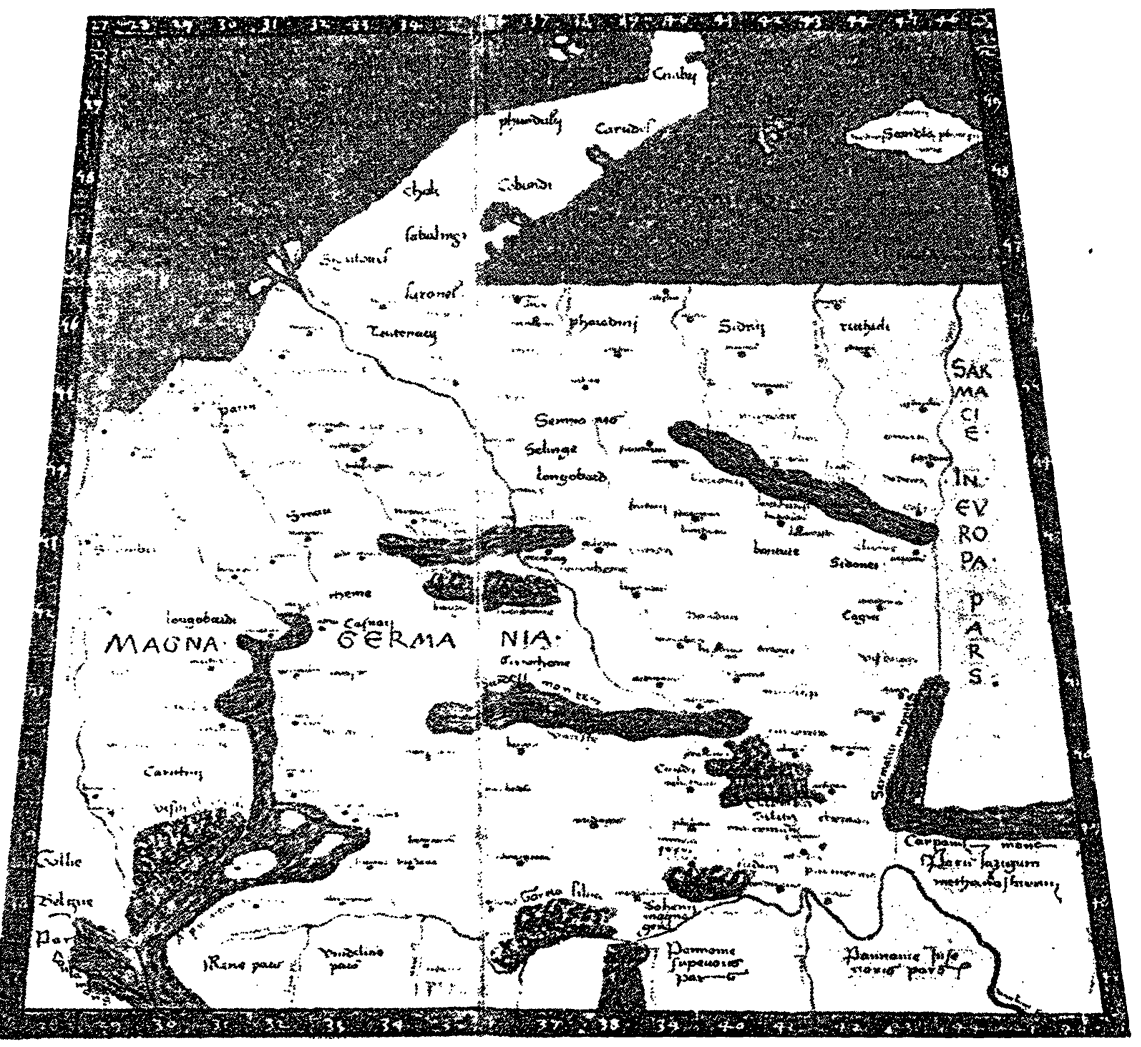


Fig. 5. VORLAGE $A^{\circ}$ - GERMANIA.

nach L. Schmidt in Seeligers "Hist. vierteljahrschrift", 1902, s. 84. Mit erlaubnis des verfassers. S. § 8 .

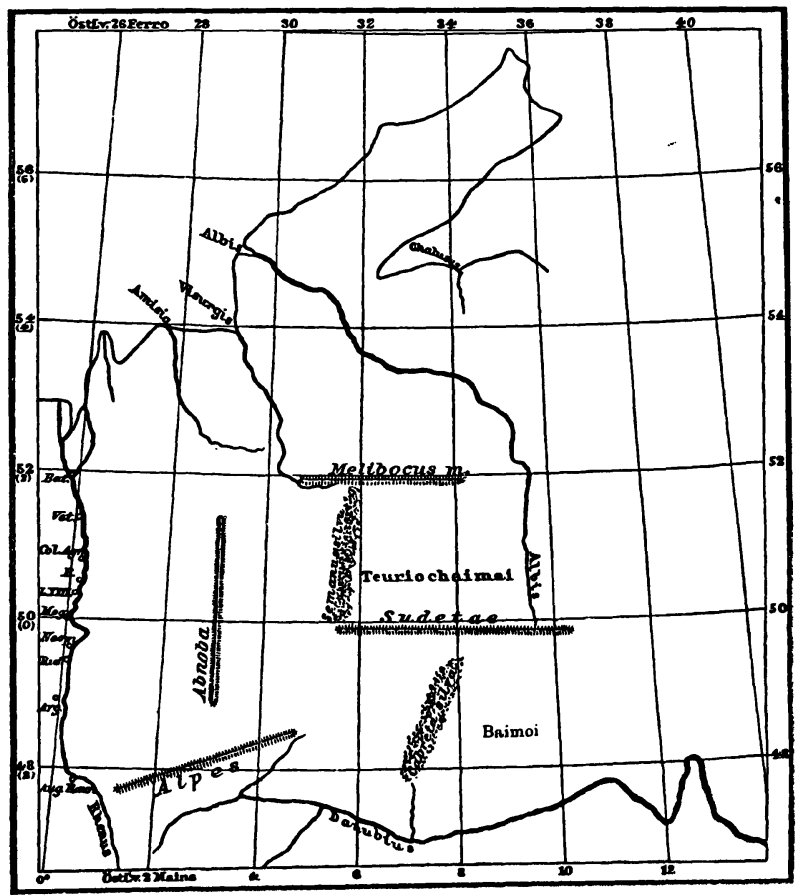




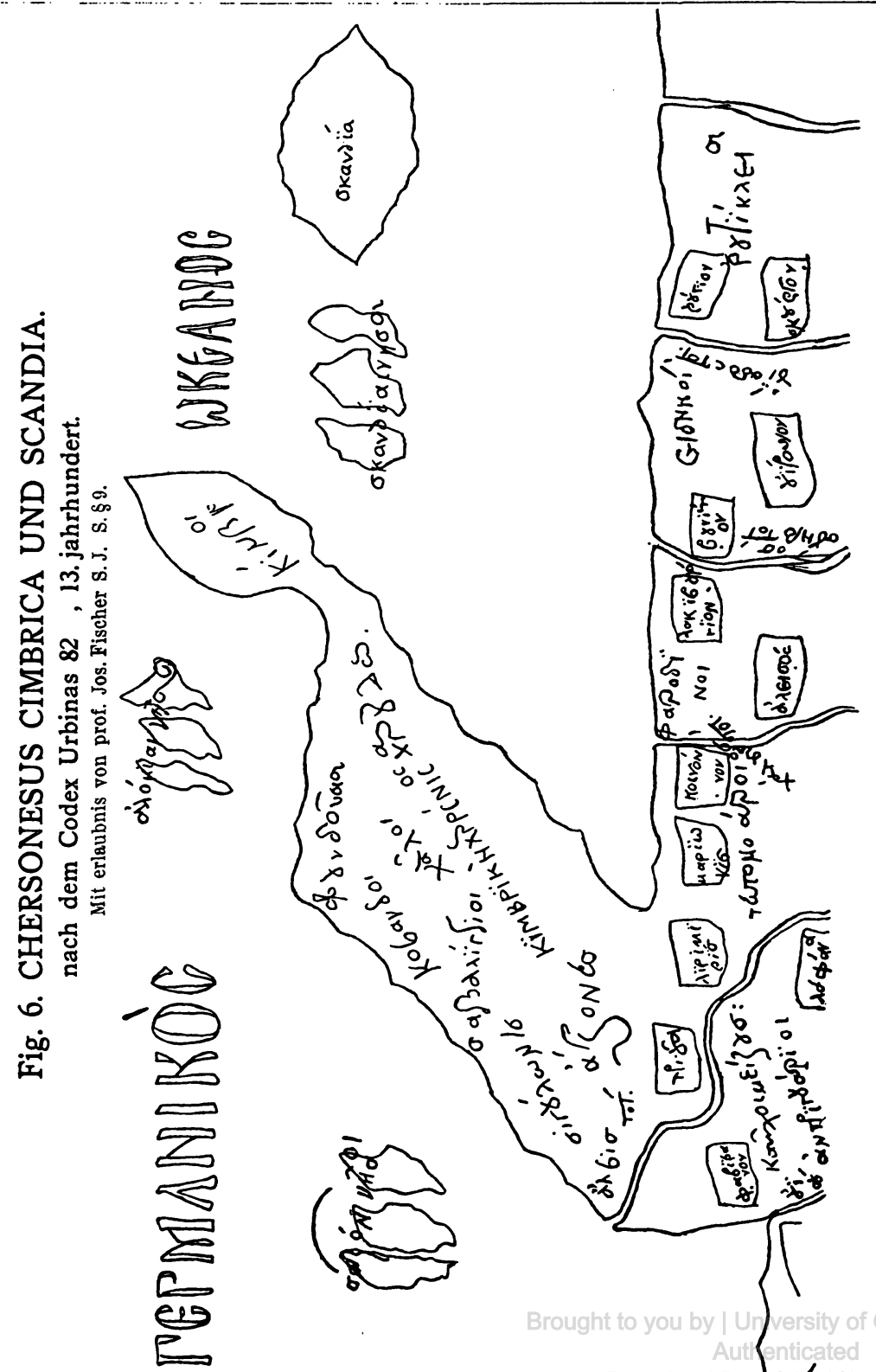




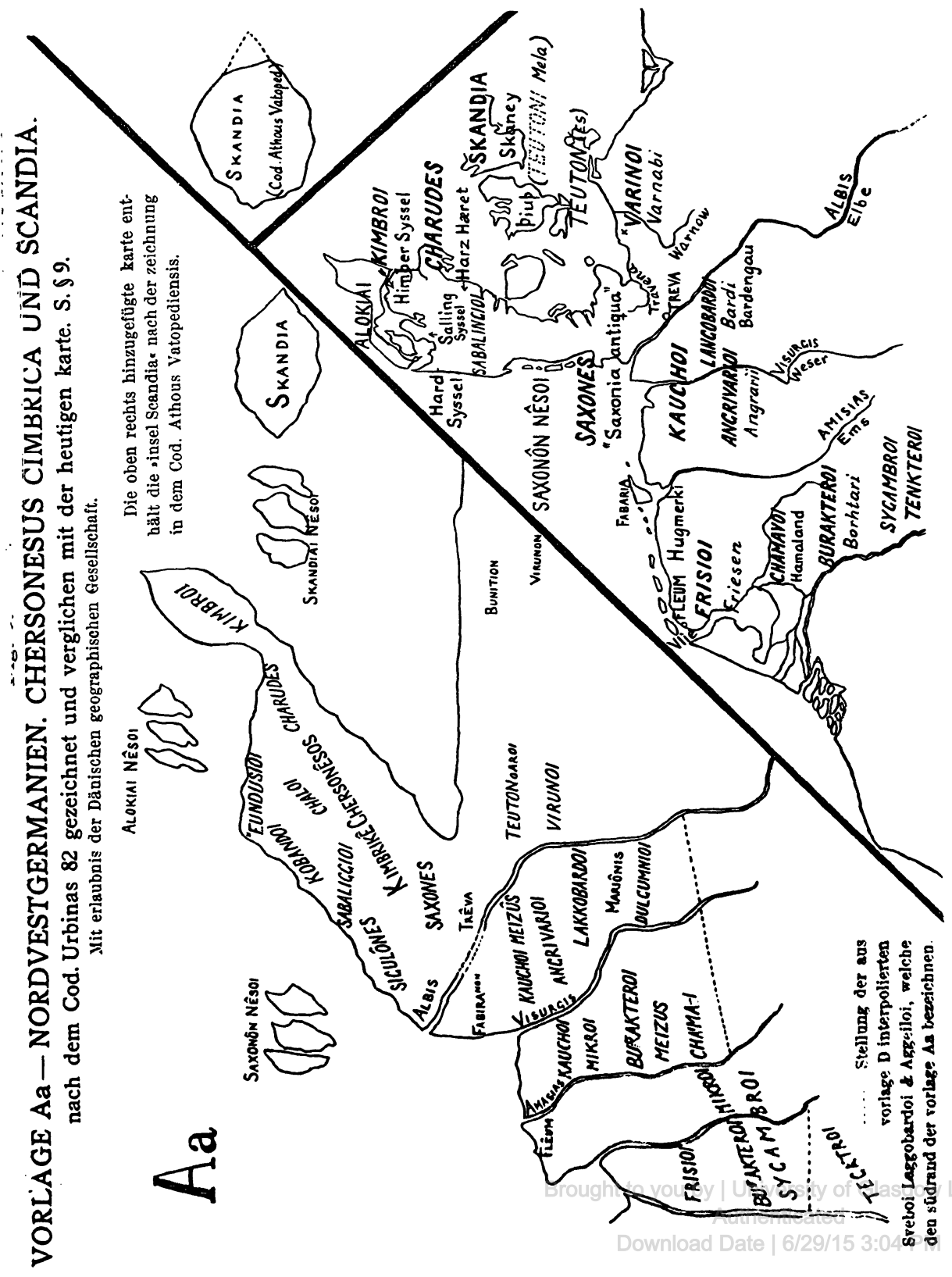


Fig. 8. VORLAGE Ab - SÜDWESTGERMANIEN, nach dem Cod. Urbinas 82 gezeichnet.

Mit erlaubuis von "The Scottish Geographical Mugazine*. S. §10.

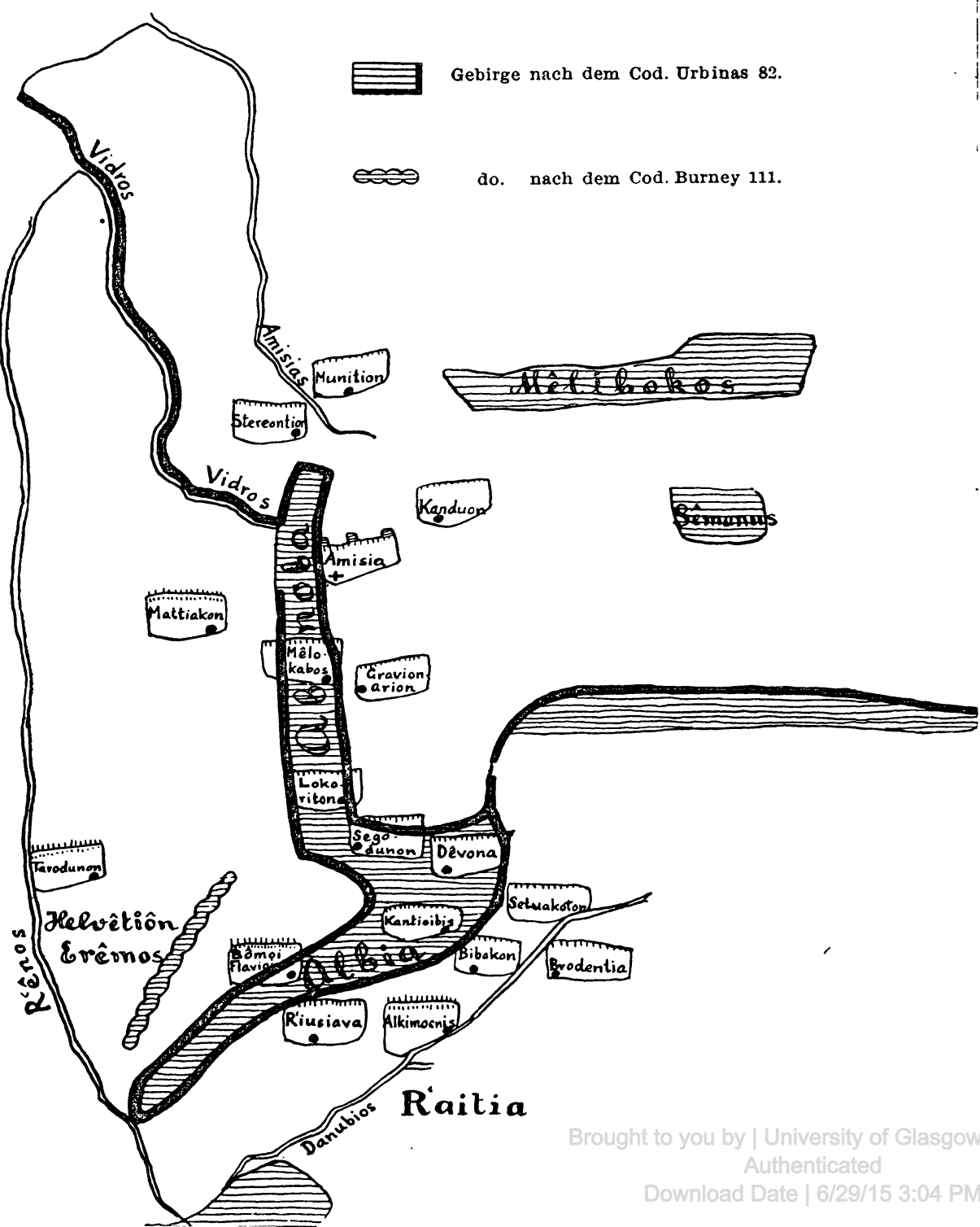


Fig. 9. LIMES TRANSRHENANUS

Mit erlaubnis von The Scottish Geographical Magazine،. S. §10.
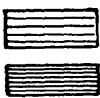

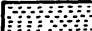

Mitteldeutsche gebirge, höhe $600-710 \mathrm{~m}$.

Süddeutsche gebirge, höhe c. $1000 \mathrm{~m}$.

Raum zwischen dem grenzwall, dem Neckar und den grenzen Rätiens, den ptolemäischen gebirgen Abnoba und Albia entsprechend.

Limes.

- Grenze der provinz Rätien.

Römische Festungen.

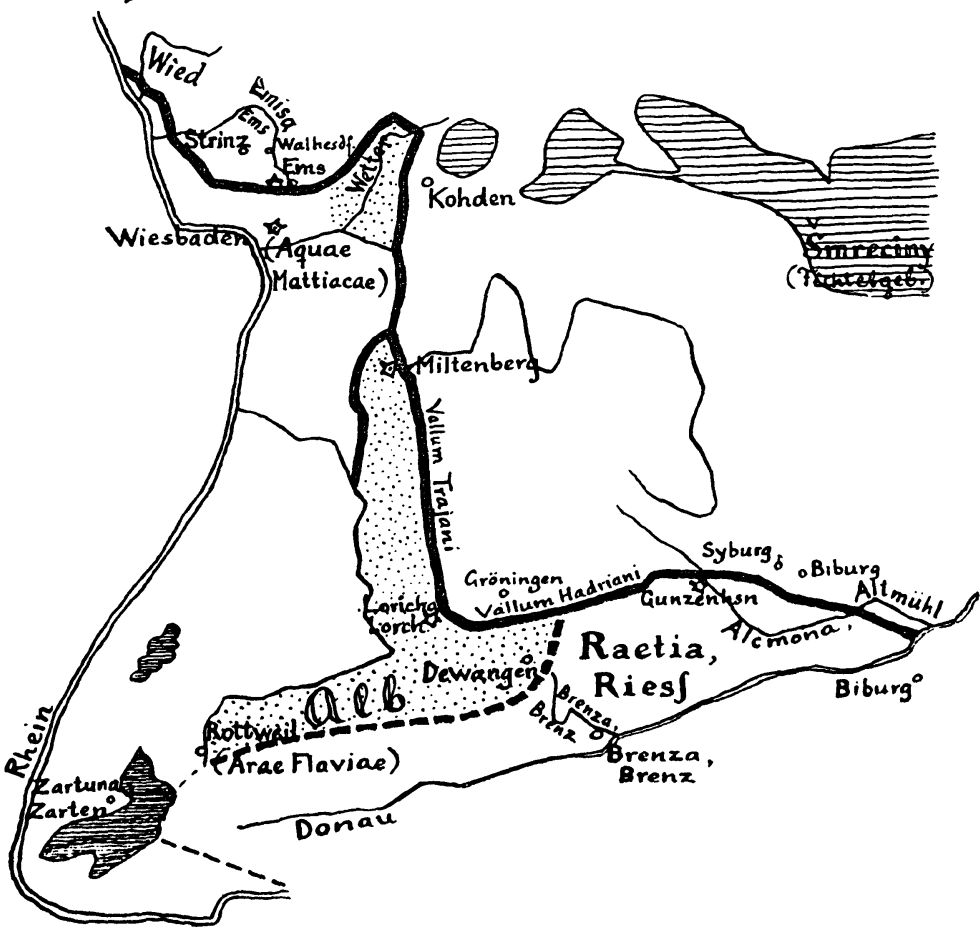




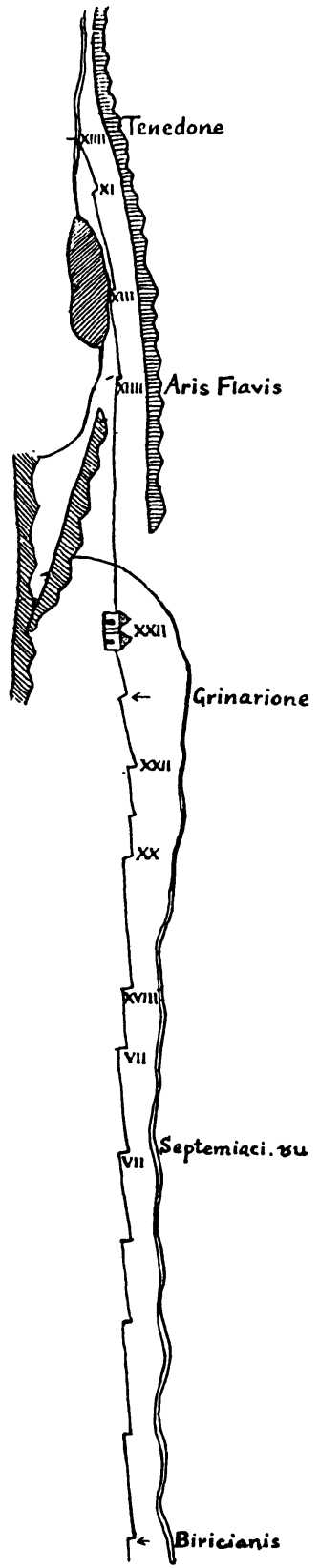

Fig. 10.

SÜDW EST GERM A N IEN nach der Tabula Peutingeriana gezeichnet. Mit erlaubnis von "The Scottish Geographical Magazine* S. § 10. 


\section{Fig. 11. VERGLEICHUNG DER EINZELHEITEN UM} DAS VALLUM HADRIANI

nach dem Cod. Urbinas 82 (A), der Tab. Peutingeriana (B) und der heutigen karte (C).

Mit erlaubnis von "The Scottish Geographical Magaziue . S. $\$ 10$.

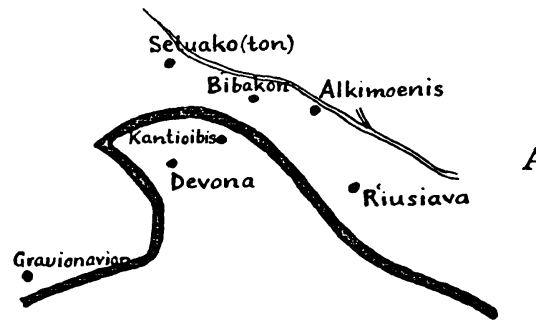

A

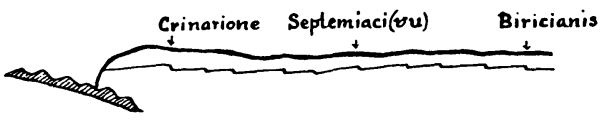

B

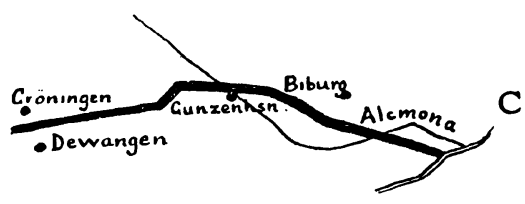




\section{Fig. 12. DAC I E N}

nach dem Codex Urbinas 82 .

Mit erlaubnis von prof. Jos. Fischer S. J. S. \$11.

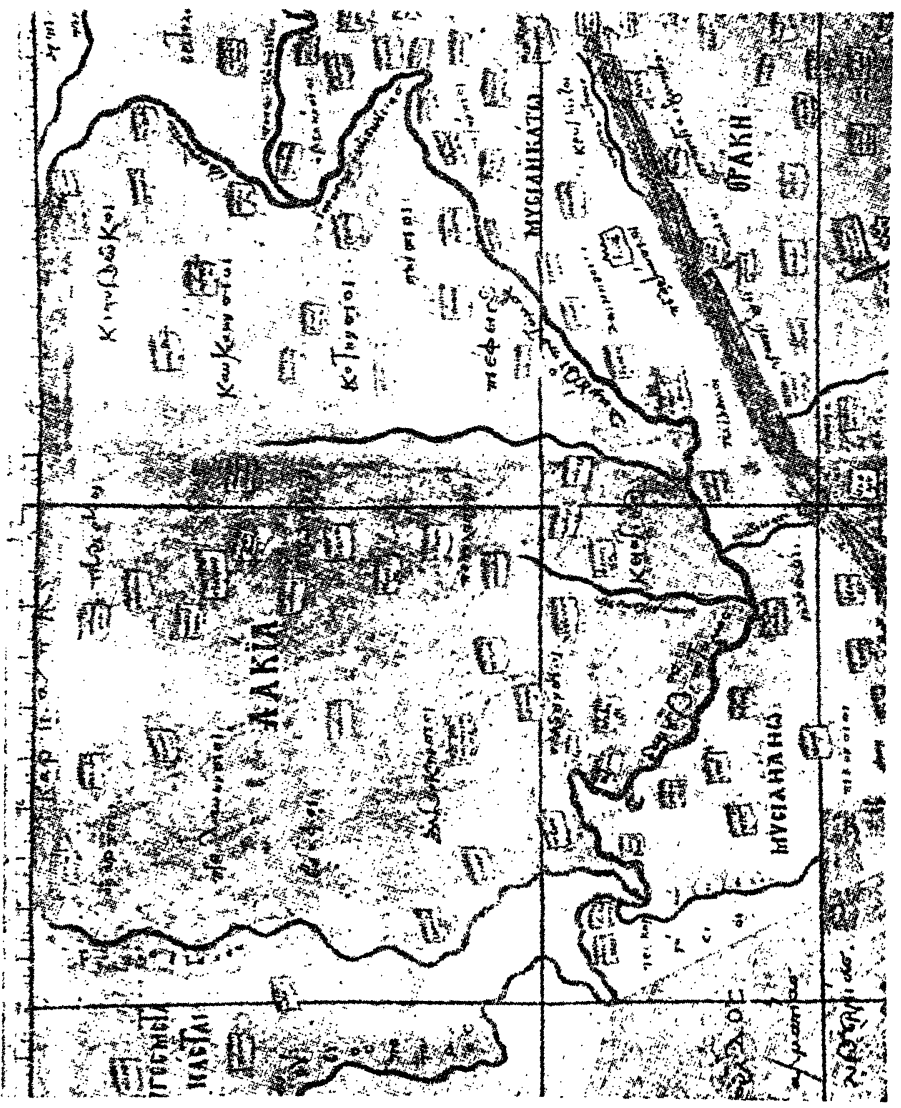


Fig. 13. VORLAGE $A c=D A C I E N$, verglichen mit der heutigen physischen karte.

Mit erlaubnis von -The Scottish Geographical Magazine*. S. § 11.

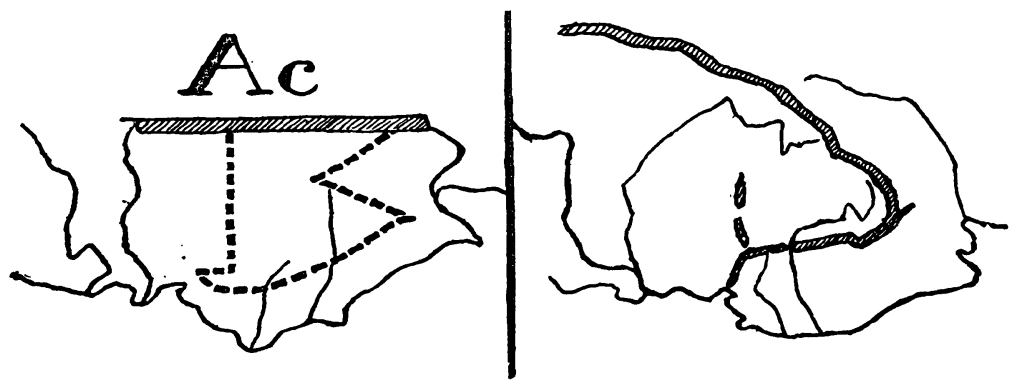

صeve. Umriss der mutmasslichen vorlage Ae, welcher von dem Ptol.-constructor mit den südlichen Karpathen der vorlage Ac identifiziert wurde. 


\section{Fig. 14. VORLAGE Ad UND Ae-DACIEN.}

\section{Vergleichung der doubletten.}

Mit erlaubnis von "The Scottish Geographical Magazine*. S.§11.

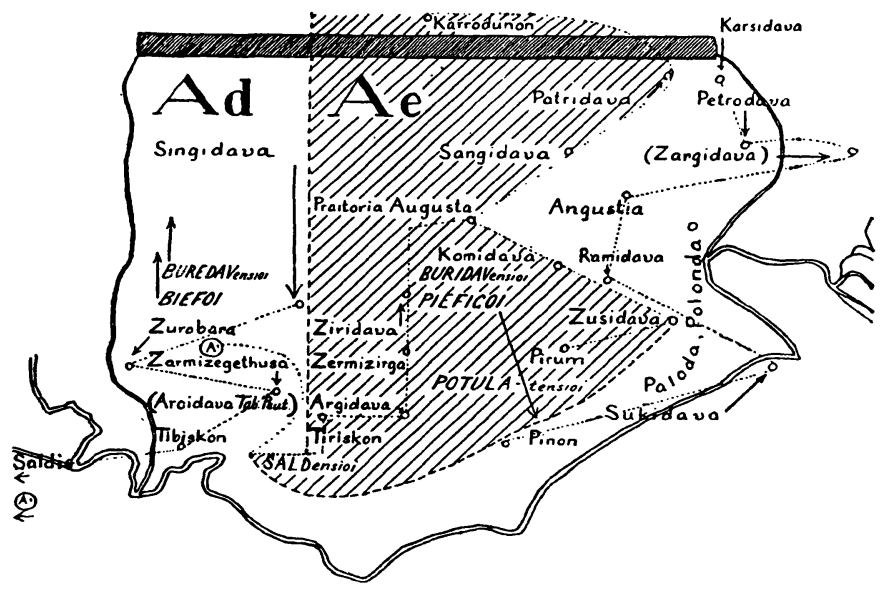

«A Richtung gegen die istrische stadt Alvona, j. All.ona, welche vielleicht zur vorlage Ad gehört.

$\ddot{A}^{\cdots}$ Platz der Albokensioi (*Albonensioi ?), welche vielleicht zur vorlage Ae gehören. 

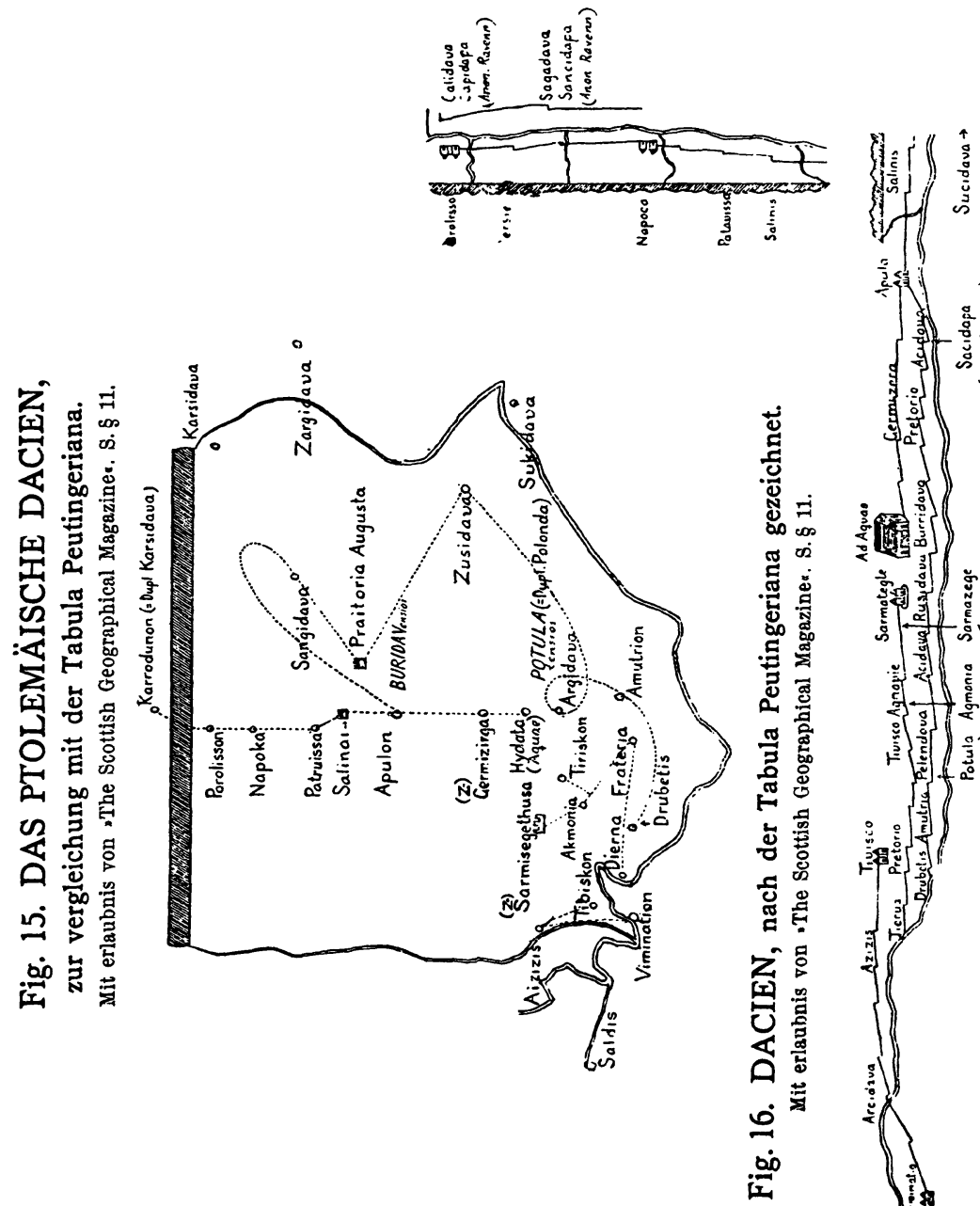

공

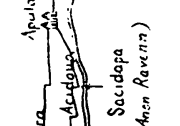

$\stackrel{9}{0}$

므

昜
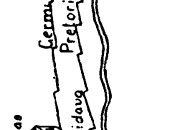

र्व

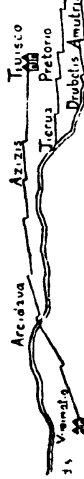

$\ddot{1}$ 


\section{Fig. 17. DAS PTOLEMÄISCHE DACIEN in die heutige karte eingezeichnet.}

Mit erlaubnis von The Scottish rieographical Magazive 4 . S. 11 .

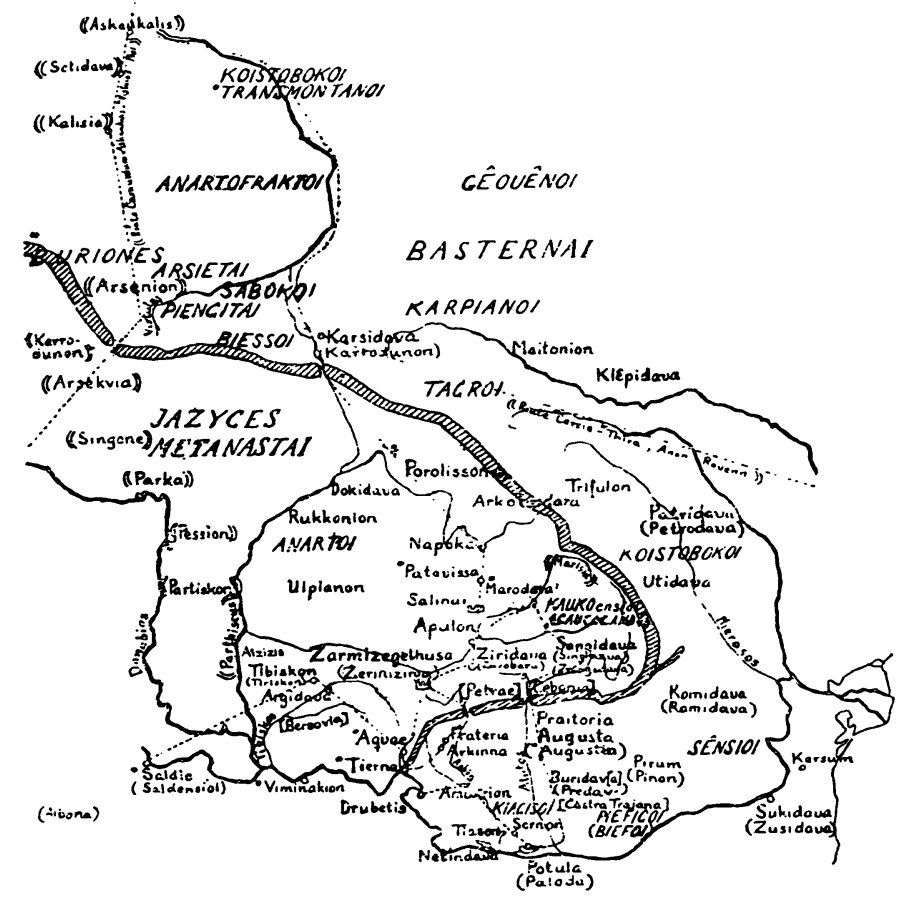

Namen ohne parenthese oder in einfacher parenthese entstammen mutmasslich den vorlagen Ac, Ad oder Ae.

Namen in zweifacher parenthese entstammen mutmasslich anderen ptolemäischen vorlagen.

Namen in eckigen klammern entstammen der Tabula Peutingeriana.

....... Handelsroute Carnuntum-Askaukalis, welche der angeblichen $*$ Weichsel- des Ptolemäus entspricht. 


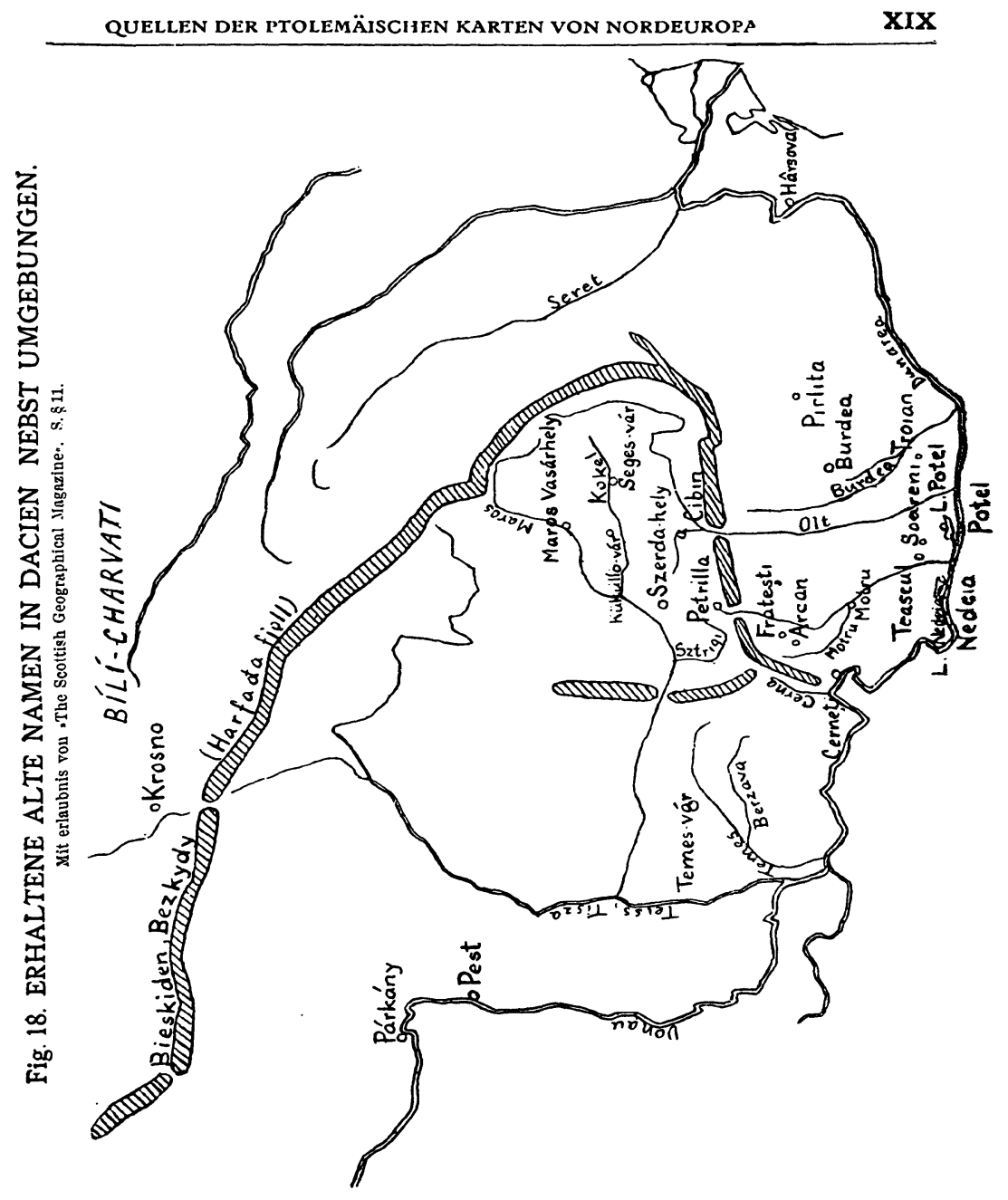


Fig. 19. VORLAGEN B1 UND B2 - OSTGERMANIEN. Vergleichung der doubletten.

Mit erlaubnis von "The Scottish Geographical Magazine*. S. § 12 .

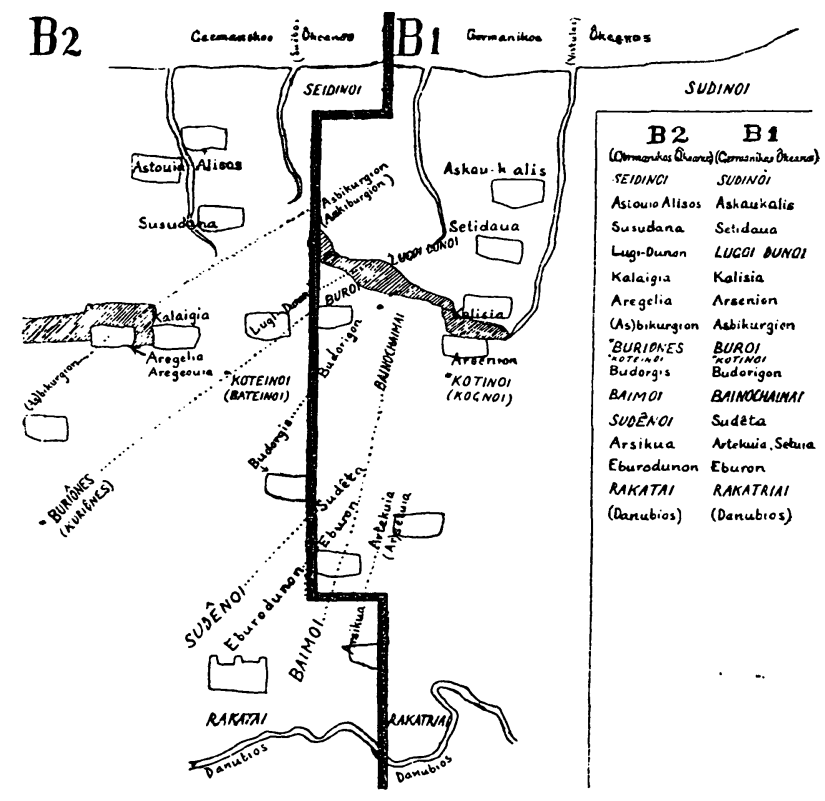


Fig. 20. VORLAGEN B1 UND B2,

verglichen mit der heutigen karte.

Mit erlaubnis von "The Scottish Geographical Magazine a. S. \& 12.

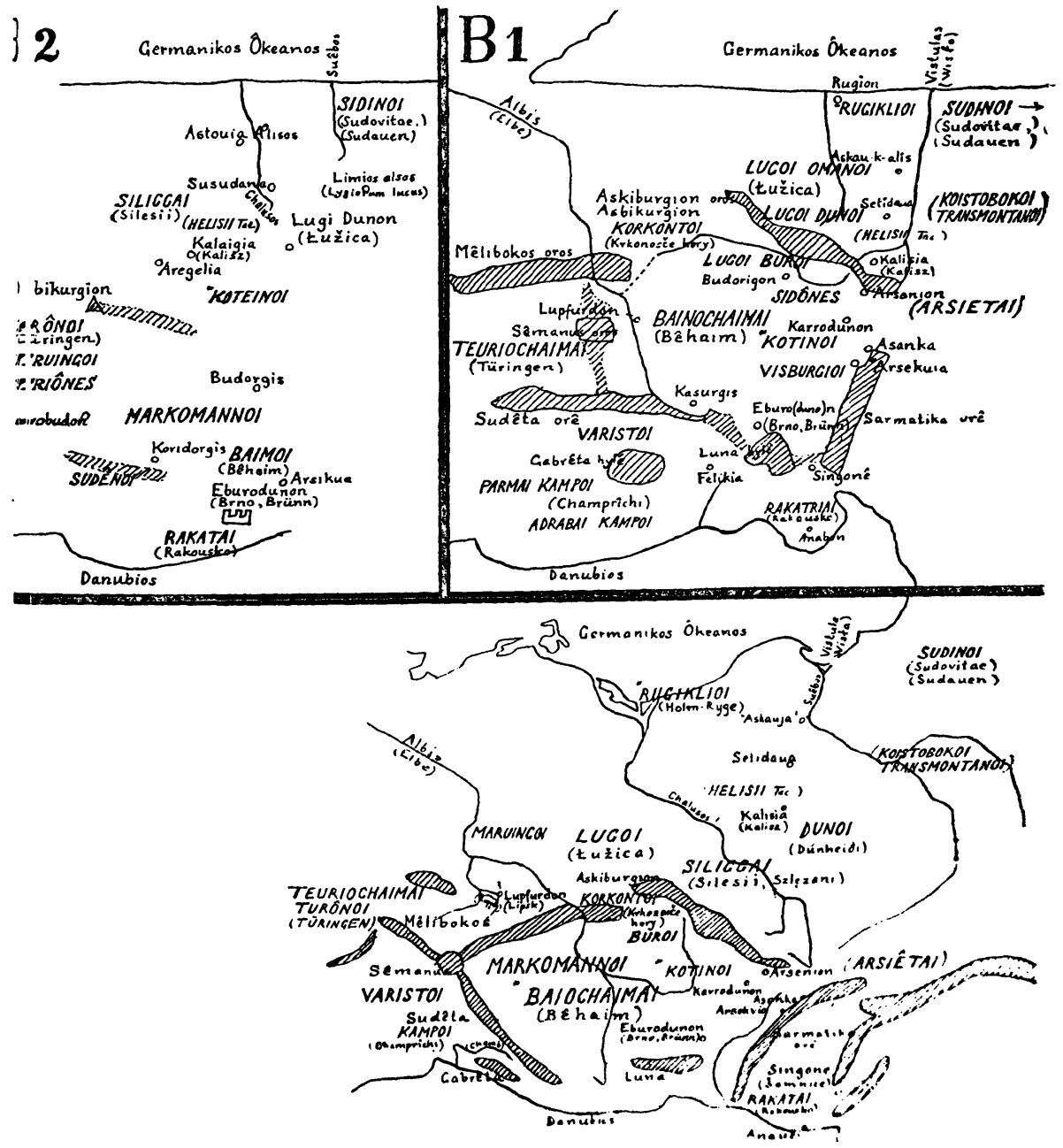

|IIIIIIIIIII (In B 1). (iebirge nneh lem (Cod. Athou V'afopedlienste.

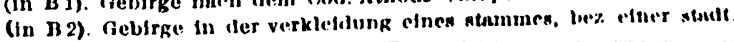




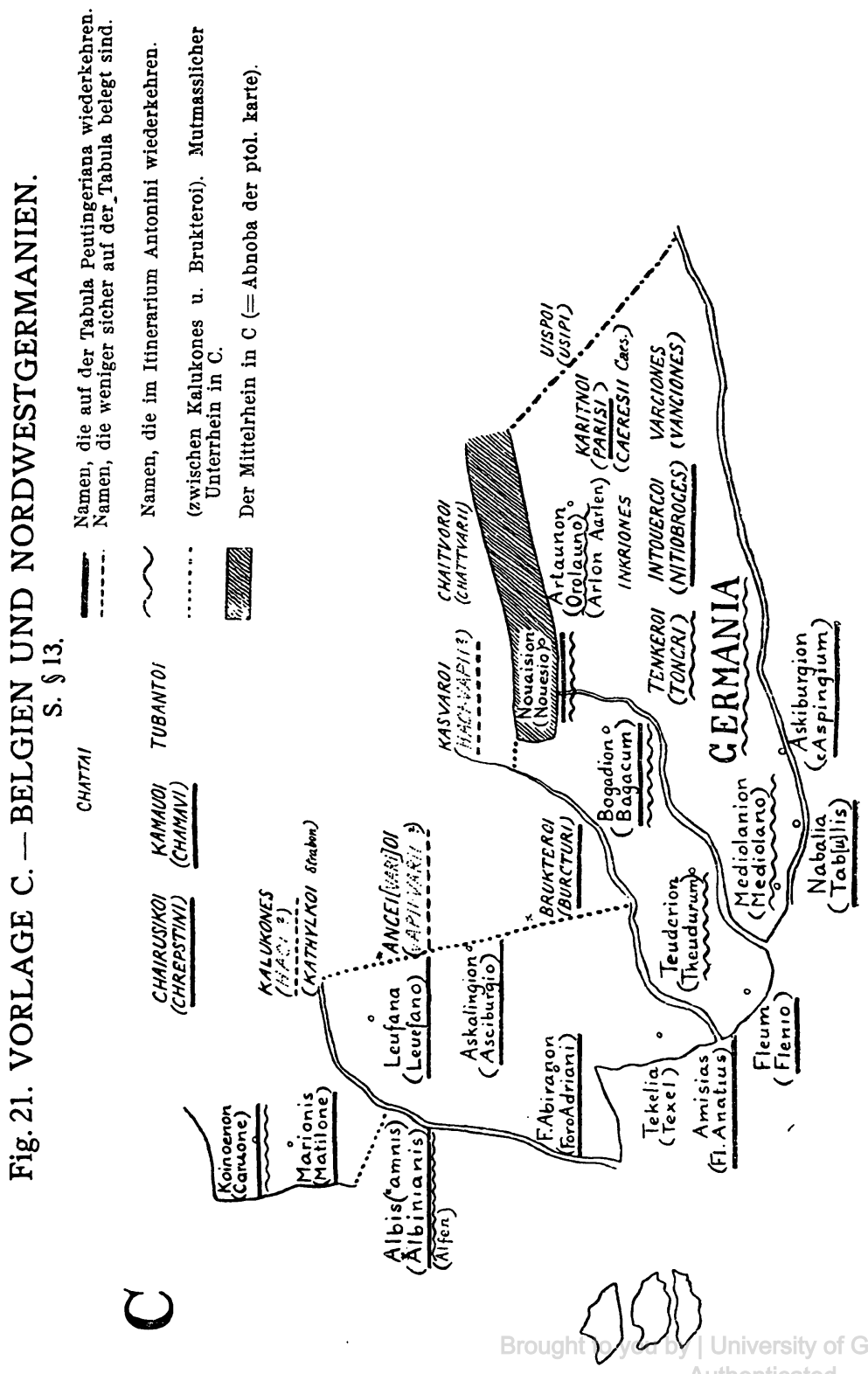




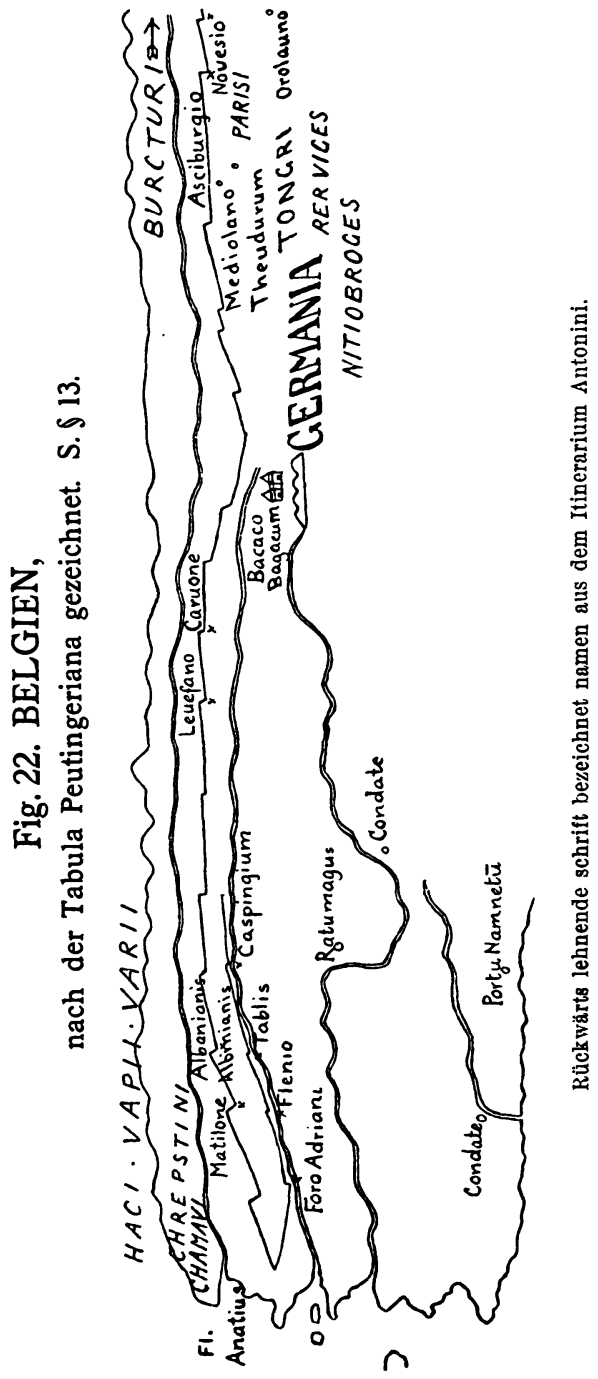


Fig. 23. DAS ANTIKE BELGIEN

UND NORDWESTGERMANIEN

mit der namenschicht der vorlage C. S. S13.

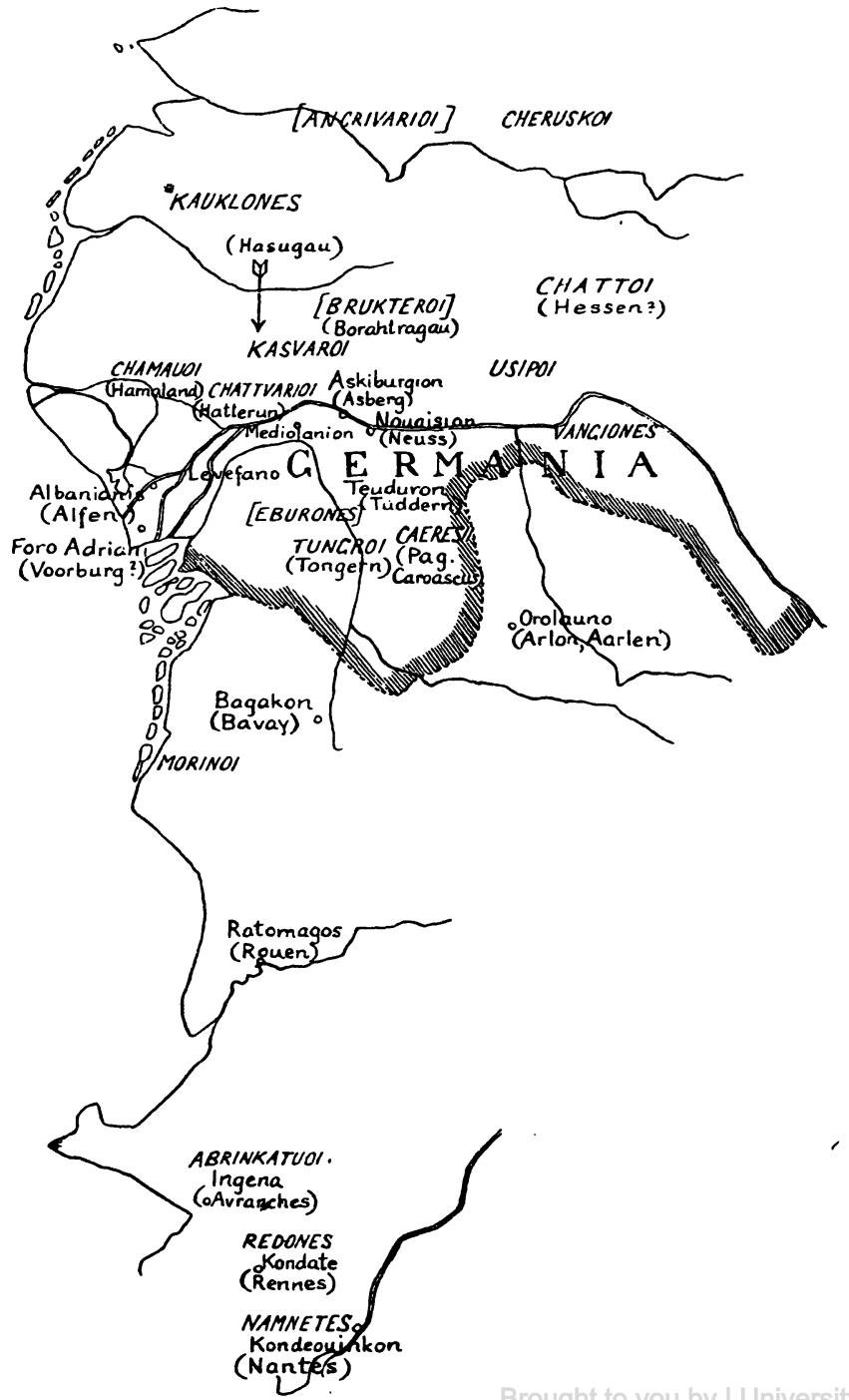




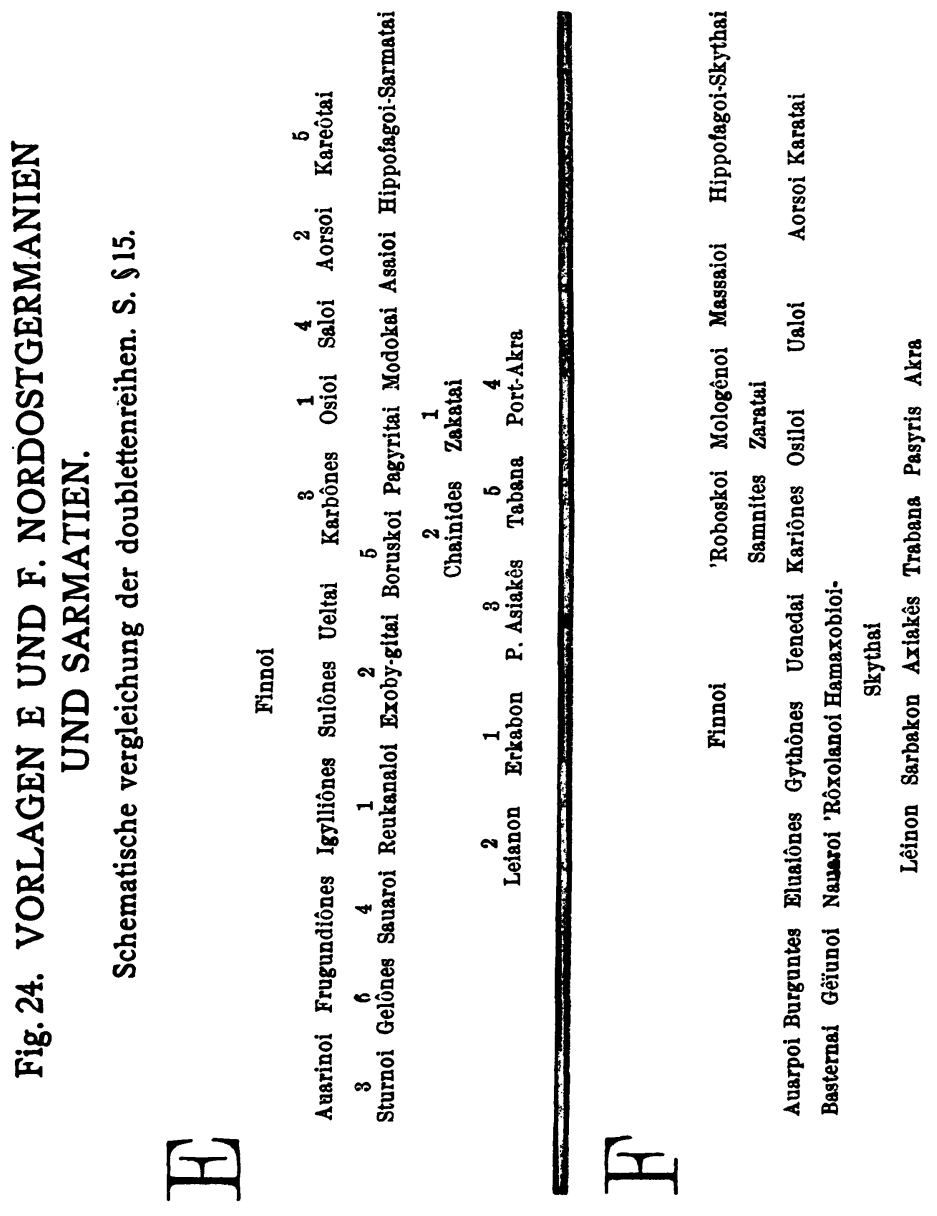


Fig. 25. VORLAGE E.

nach dem Cod. Urbinas 82 gezeichnet.

Mit erlaubnis von -The Scottish Geographical Magazine*. S. §15.

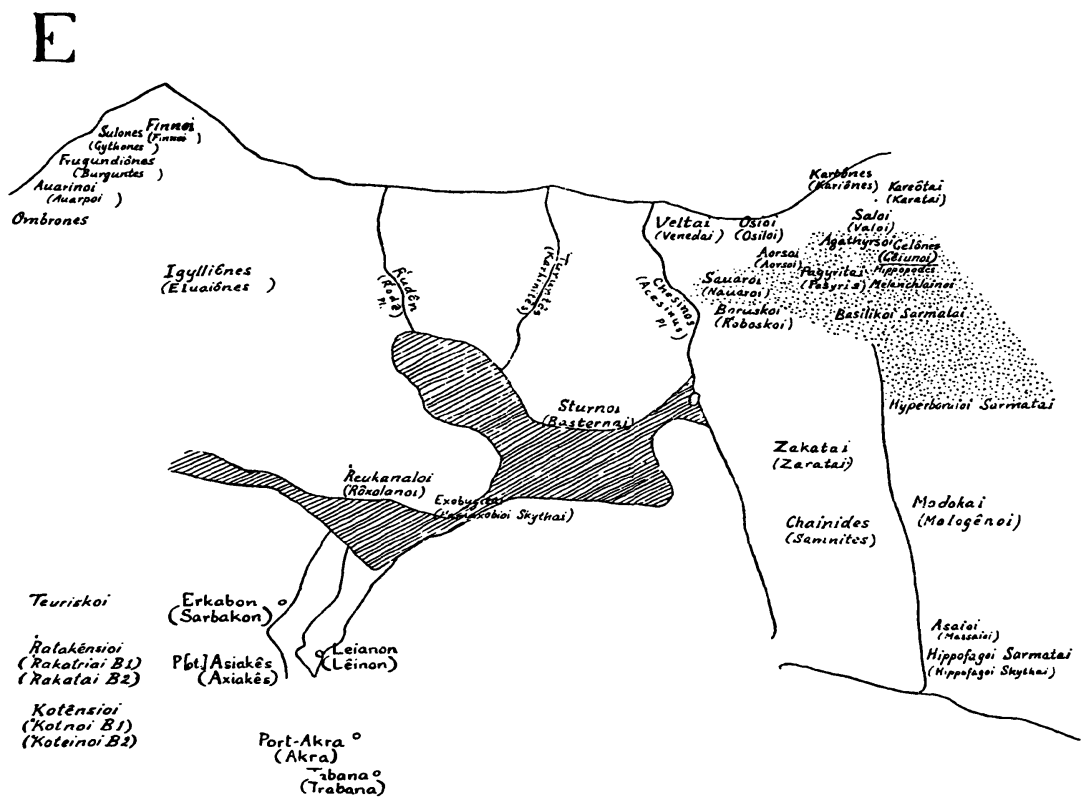

Namen in parenthese bezeichnen entsprechungen aus $F$.

Teilweise veraltete namen aus Herodot.

|!|||||||||||| Gebirge. 
Fig. 26.

\section{NORDOSTGERMANIEN UND WESTSARMATIEN}

mit den namenschichten der vorlagen $E$ und $F$.

S. § 15

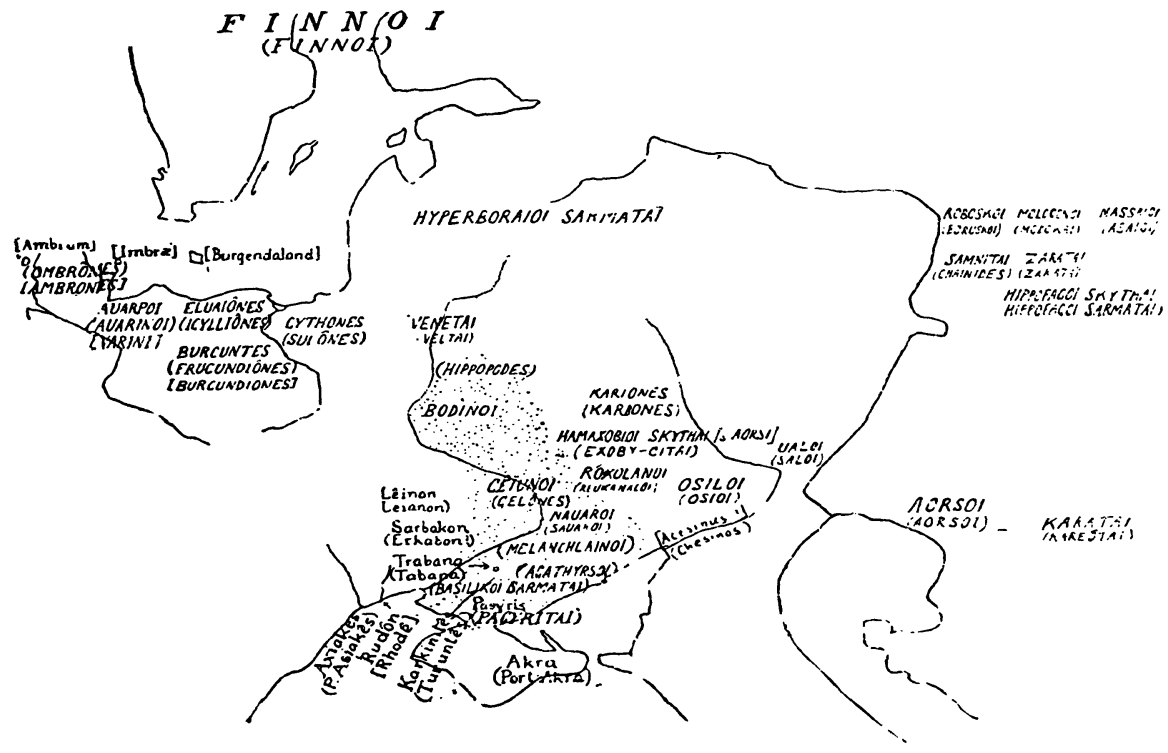

$N A M E N$ in parenthese gebörep der vorlage $\mathbf{E}$.

$N A M E N$ ohne parenthese gehören der vorlage $F$.

Nameu in eckigen klammern sind aus anderen Quellen (zumal Plinius) zur ver. gleichuvg hinzugefügt.

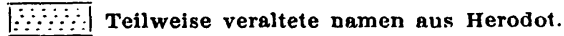


Fig. 27. VORLAGE SK - SCANDIA.

S. $\$ 16$.
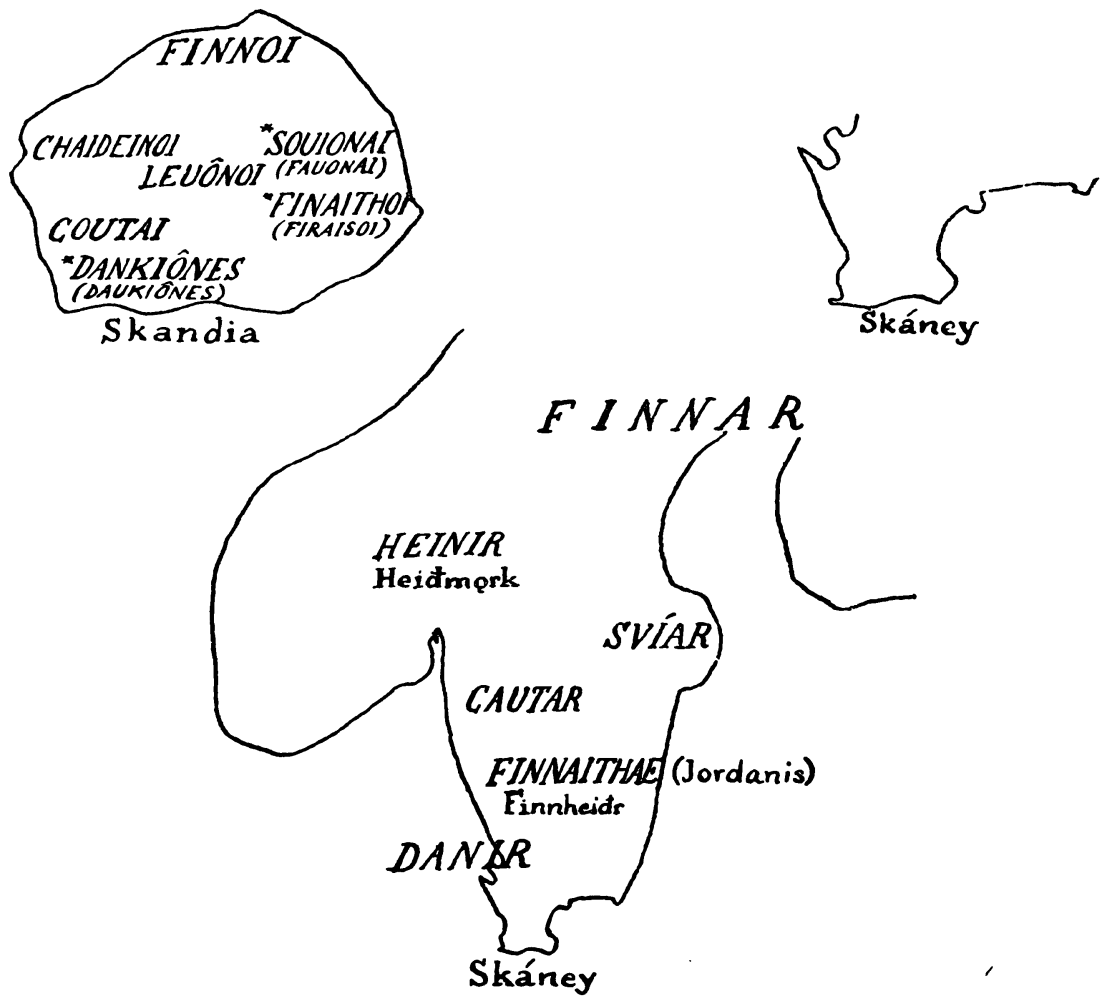
Fig. 28.

\section{GERMANIA, CHERSONESUS CIMBRICA, SCANDIA}

nach dem Codex Athous Vatopediensis, 13. jahrhundert.

Mit erlaubnis der Ininischen geographis-hen (iecellschaft.

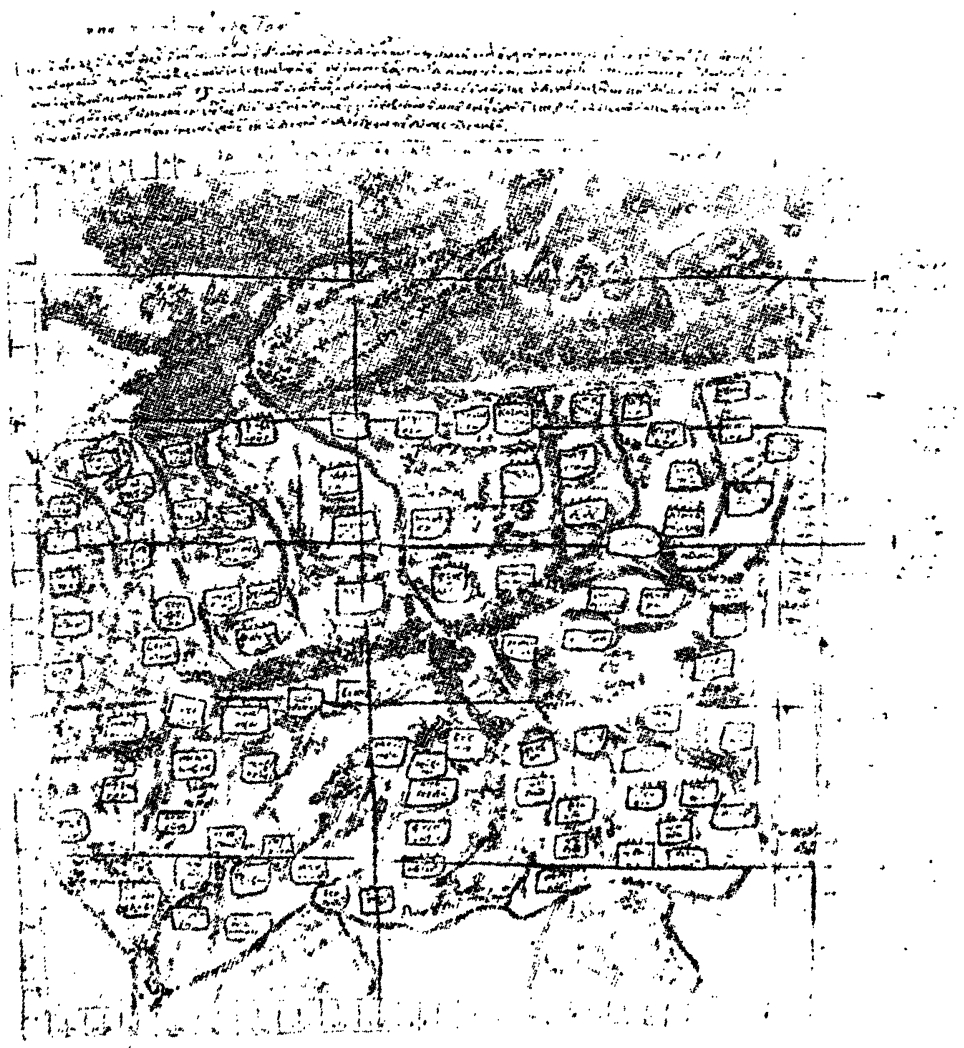

